\title{
A CHANGE OF RINGS RESULT FOR MATLIS REFLEXIVITY
}

\author{
DOUGLAS J. DAILEY AND THOMAS MARLEY
}

(Communicated by Irena Peeva)

\begin{abstract}
Let $R$ be a commutative Noetherian ring and $E$ the minimal injective cogenerator of the category of $R$-modules. An $R$-module $M$ is (Matlis) reflexive if the natural evaluation map $M \longrightarrow \operatorname{Hom}_{R}\left(\operatorname{Hom}_{R}(M, E), E\right)$ is an isomorphism. We prove that if $S$ is a multiplicatively closed subset of $R$ and $M$ is an $R_{S}$-module which is reflexive as an $R$-module, then $M$ is a reflexive $R_{S}$-module. The converse holds when $S$ is the complement of the union of finitely many nonminimal primes of $R$, but fails in general.
\end{abstract}

\section{INTRODUCTION}

Let $R$ be a commutative Noetherian ring and $E$ the minimal injective cogenerator of the category of $R$-modules; i.e., $E=\bigoplus_{m \in \Lambda} E_{R}(R / m)$, where $\Lambda$ denotes the set of maximal ideals of $R$ and $E_{R}(-)$ denotes the injective hull. An $R$-module $M$ is said to be (Matlis) reflexive if the natural evaluation map $M \longrightarrow \operatorname{Hom}_{R}\left(\operatorname{Hom}_{R}(M, E), E\right)$ is an isomorphism. In [1, the authors assert the following "change of rings" principal for Matlis reflexivity ([1, Lemma 2]): Let $S$ be a multiplicatively closed subset of $R$ and suppose $M$ is an $R_{S}$-module. Then $M$ is reflexive as an $R$-module if and only if $M$ is reflexive as an $R_{S}$-module. However, the proof given in [1] contains an error (see Examples 3.1-3.3), and in fact the "if" part is false in general (cf. Proposition 3.4). In this note, we prove the following:

Theorem 1.1. Let $R$ be a Noetherian ring, $S$ a multiplicatively closed subset of $R$, and $M$ an $R_{S}$-module.

(a) If $M$ is reflexive as an $R$-module, then $M$ is reflexive as an $R_{S}$-module.

(b) If $S=R \backslash\left(p_{1} \cup \cdots \cup p_{r}\right)$ where each $p_{i}$ is a maximal ideal or a nonminimal prime ideal, then the converse to (a) holds.

\section{MAin RESUlts}

Throughout this section $R$ will denote a Noetherian ring and $S$ a multiplicatively closed subset of $R$. We let $E_{R}$ (or just $E$ if the ring is clear) denote the minimal injective cogenerator of the category of $R$-modules as defined in the introduction. A semilocal ring is said to be complete if it is complete with respect to the $J$-adic topology, where $J$ is the Jacobson radical.

Received by the editors October 14, 2015 and, in revised form, May 11, 2016.

2010 Mathematics Subject Classification. Primary 13C05; Secondary 13C13.

Key words and phrases. Matlis reflexive, minimal injective cogenerator.

The first author was partially supported by U.S. Department of Education grant P00A120068 (GAANN). 
We will make use of the main result of [1]:

Theorem 2.1 ([1, Theorem 12]). Let $R$ be a Noetherian ring, $M$ an $R$-module, and $I=\operatorname{Ann}_{R} M$. Then $M$ is reflexive if and only if $R / I$ is a complete semilocal ring and there exists a finitely generated submodule $N$ of $M$ such that $M / N$ is Artinian.

We remark that the validity of this theorem does not depend on [1, Lemma 2], as the proof of [1, Theorem 12] uses this lemma only in a special case where it is easily seen to hold. (See the proof of [1, Theorem 9], which is the only instance where [1, Lemma 2] is used critically.)

Lemma 2.2 ([1, Lemma 1]). Let $M$ be an $R$-module and $I$ an ideal of $R$ such that $I M=0$. Then $M$ is reflexive as an $R$-module if and only if $M$ is reflexive as an $R / I$-module.

Proof. Since $E_{R / I}=\operatorname{Hom}_{R}\left(R / I, E_{R}\right)$, the result follows readily by Hom-tensor adjunction.

Lemma 2.3. Let $R=R_{1} \times \cdots \times R_{k}$ be a product of Noetherian local rings. Let $M=M_{1} \times \cdots \times M_{k}$ be an $R$-module. Then $M$ is reflexive as an $R$-module if and only if $M_{i}$ is reflexive as an $R_{i}$-module for all $i$.

Proof. As finite sums and direct summands of reflexive modules are reflexive, it suffices to prove that $M_{i}$ is reflexive as an $R$-module if and only if $M_{i}$ is reflexive as an $R_{i}$-module for each $i$. But this follows immediately from Lemma 2.2

Theorem 2.4. Let $S$ be a multiplicatively closed subset of $R$ and $M$ an $R_{S}$-module which is reflexive as an $R$-module. Then $M$ is reflexive as an $R_{S}$-module.

Proof. By Lemma 2.2, we may assume $\operatorname{Ann}_{R} M=\operatorname{Ann}_{R_{S}} M=0$. Thus, $R$ is semilocal and complete by Theorem 2.1. Hence, $R=R_{1} \times \cdots \times R_{k}$ where each $R_{i}$ is a complete local ring. Then $R_{S}=\left(R_{1}\right)_{S_{1}} \times \cdots \times\left(R_{k}\right)_{S_{k}}$ where $S_{i}$ is the image of $S$ under the canonical projection $R \longrightarrow R_{i}$. Write $M=M_{1} \times \cdots \times M_{k}$, where $M_{i}=R_{i} M$. As $M$ is reflexive as an $R$-module, $M_{i}$ is reflexive as an $R_{i}$-module for all $i$. Thus, it suffices to show that $M_{i}$ is reflexive as an $\left(R_{i}\right)_{S_{i}}$-module for all $i$. Hence, we may reduce the proof to the case where $(R, m)$ is a complete local ring with $\operatorname{Ann}_{R} M=0$ by passing to $R / \operatorname{Ann}_{R} M$, if necessary. As $M$ is reflexive as an $R$-module, we have by Theorem 2.1 that there exists an exact sequence

$$
0 \longrightarrow N \longrightarrow M \longrightarrow X \longrightarrow 0
$$

where $N$ is a finitely generated $R$-module and $X$ is an Artinian $R$-module. If $S \cap m=$ $\emptyset$, then $R_{S}=R$ and there is nothing to prove. Otherwise, as $\operatorname{Supp}_{R} X \subseteq\{m\}$, we have $X_{S}=0$. Hence, $M \cong N_{S}$, a finitely generated $R_{S}$-module. To see that $M$ is $R_{S}$-reflexive, it suffices to show that $R_{S}$ is Artinian (hence semilocal and complete). Since $\operatorname{Ann}_{R} N_{S}=\operatorname{Ann}_{R} M=0$, we have that $\operatorname{Ann}_{R} N=0$. Thus, $\operatorname{dim} R=\operatorname{dim} N$. Since $M$ is an $R_{S}$-module and $S \cap m \neq \emptyset$, we have $H_{m}^{i}(M) \cong H_{m R_{S}}^{i}(M)=0$ for all $i$. Further, as $X$ is Artinian, $H_{m}^{i}(X)=0$ for $i \geq 1$. Thus, from the long exact sequence on local cohomology, we conclude that $H_{m}^{i}(N)=0$ for $i \geq 2$. Thus, $\operatorname{dim} R=\operatorname{dim} N \leq 1$, and hence, $\operatorname{dim} R_{S}=0$. Consequently, $R_{S}$ is Artinian, and $M$ is a reflexive $R_{S}$-module.

To prove part (b) of Theorem 1.1, we will need the following result on Henselian local rings found in 2] (in which the authors credit it to F. Schmidt). As we need 
a slightly different version of this result than what is stated in [2] and the proof is short, we include it for the convenience of the reader:

Proposition 2.5 ([2, Satz 2.3.11]). Let $(R, m)$ be a local Henselian domain which is not a field and $F$ the field of fractions of $R$. Let $V$ be a discrete valuation ring with field of fractions $F$. Then $R \subseteq V$.

Proof. Let $k$ be the residue field of $R$ and $a \in m$. As $R$ is Henselian, for every positive integer $n$ not divisible by the characteristic of $k$, the polynomial $x^{n}-(1+a)$ has a root $b$ in $R$. Let $v$ be the valuation on $F$ associated to $V$. Then $n v(b)=$ $v(1+a)$. If $v(a)<0$, then $v(1+a)<0$, which implies that $v(b) \leq-1$. Hence, $v(1+a) \leq-n$. As $n$ can be arbitrarily large, this leads to a contradiction. Hence, $v(a) \geq 0$ and $a \in V$. Thus, $m \subseteq V$. Now let $c \in R$ be arbitrary. Choose $d \in m, d \neq 0$. If $v(c)<0$, then $v\left(c^{\ell} d\right)<0$ for $\ell$ sufficiently large. But this contradicts that $c^{\ell} d \in m \subseteq V$ for every $\ell$. Hence $v(c) \geq 0$ and $R \subseteq V$.

For a Noetherian ring $R$, let $\operatorname{Min} R$ and $\operatorname{Max} R$ denote the set of minimal and maximal primes of $R$, respectively. Let $\mathrm{T}(R)=(\operatorname{Spec} R \backslash \operatorname{Min} R) \cup \operatorname{Max} R$.

Lemma 2.6. Let $R$ be a Noetherian ring and $p \in \mathrm{T}(R)$. If $R_{p}$ is Henselian, then the natural map $\varphi: R \longrightarrow R_{p}$ is surjective; i.e., $R / \operatorname{ker} \varphi \cong R_{p}$.

Proof. By replacing $R$ with $R / \operatorname{ker} \varphi$, we may assume $\varphi$ is injective. Then $p$ contains every minimal prime of $R$. Let $u \in R, u \notin p$. It suffices to prove that the image of $u$ in $R / q$ is a unit for every minimal prime $q$ of $R$. Hence, we may assume that $R$ is a domain. (Note that $(R / q)_{p}=R_{p} / q R_{p}$ is still Henselian.) If $R_{p}$ is a field, then, as $p \in \mathrm{T}(R)$, we must have that $R$ is a field (as $p$ must be both minimal and maximal in a domain). So certainly $u \notin p=(0)$ is a unit in $R$. Thus, we may assume $R_{p}$ is not a field. Suppose $u$ is not a unit in $R$. Then $u \in n$ for some maximal ideal $n$ of $R$. Now, there exists a discrete valuation ring $V$ with the same field of fractions as $R$ such that $m_{V} \cap R=n$ ([3, Theorem 6.3.3]). As $R_{p}$ is Henselian, $R_{p} \subseteq V$ by Proposition 2.5. But as $u \notin p, u$ is a unit in $R_{p}$, hence in $V$, contradicting $u \in n \subseteq m_{V}$. Thus, $u$ is a unit in $R$ and $R=R_{p}$.

Proposition 2.7. Let $R$ be a Noetherian ring and $S=R \backslash\left(p_{1} \cup \cdots \cup p_{r}\right)$ where $p_{1}, \ldots, p_{r} \in \mathrm{T}(R)$. Suppose $R_{S}$ is complete with respect to its Jacobson radical. Then the natural map $\varphi: R \longrightarrow R_{S}$ is surjective.

Proof. First, we may assume that $p_{j} \nsubseteq \bigcup_{i \neq j} p_{i}$ for all $j$. Also, by passing to the ring $R / \operatorname{ker} \varphi$, we may assume $\varphi$ is injective. (We note that if $p_{i_{1}}, \ldots, p_{i_{t}}$ are the ideals in the set $\left\{p_{1}, \ldots, p_{r}\right\}$ containing $\operatorname{ker} \varphi$, it is easily seen that $(R / \operatorname{ker} \varphi)_{S}=(R / \operatorname{ker} \varphi)_{T}$ where $T=R \backslash\left(p_{i_{1}} \cup \cdots \cup p_{i_{t}}\right)$. Hence, we may assume each $p_{i}$ contains $\operatorname{ker} \varphi$.) As $R_{S}$ is semilocal and complete, the map $\psi: R_{S} \longrightarrow R_{p_{1}} \times \cdots \times R_{p_{r}}$ given by $\psi(u)=\left(\frac{u}{1}, \ldots, \frac{u}{1}\right)$ is an isomorphism. For each $i$, let $\rho_{i}: R \longrightarrow R_{p_{i}}$ be the natural map. Since $R \longrightarrow R_{S}$ is an injection, $\bigcap_{i} \operatorname{ker} \rho_{i}=(0)$. It suffices to prove that $u$ is a unit in $R$ for every $u \in S$. As $R_{p_{i}}$ is complete, hence Henselian, we have that $\rho_{i}$ is surjective for each $i$ by Lemma 2.6. Thus, $u$ is a unit in $R / \operatorname{ker} \rho_{i}$ for every $i$; i.e., $(u)+\operatorname{ker} \rho_{i}=R$ for $i=1, \ldots, r$. Then $(u)=(u)+\left(\bigcap_{i} \operatorname{ker} \rho_{i}\right)=R$. Hence, $u$ is a unit in $R$. 
We now prove part (b) of the Theorem 1.1

Theorem 2.8. Let $R$ be a Noetherian ring and $M$ a reflexive $R_{S}$-module, where $S$ is the complement in $R$ of the union of finitely many elements of $\mathrm{T}(R)$. Then $M$ is reflexive as an $R$-module.

Proof. We may assume $M \neq 0$. Let $S=R \backslash\left(p_{1} \cup \cdots \cup p_{r}\right)$, where $p_{1}, \ldots, p_{r} \in \mathrm{T}(R)$. Let $I=\operatorname{Ann}_{R} M$, whence $I_{S}=\operatorname{Ann}_{R_{S}} M$. As in the proof of Proposition 2.7 we may assume each $p_{i}$ contains $I$. Then by Lemma 2.2. we may reduce to the case $\operatorname{Ann}_{R} M=\operatorname{Ann}_{R_{S}} M=0$. Note that this implies that the natural map $R \longrightarrow R_{S}$ is injective. As $M$ is $R_{S}$-reflexive, $R_{S}$ is complete with respect to its Jacobson radical by Theorem 2.1. By Proposition 2.7, we have that $R \cong R_{S}$ and hence $M$ is $R$-reflexive.

\section{EXAMPLES}

The following examples show that $\operatorname{Hom}_{R}\left(R_{S}, E_{R}\right)$ need not be the minimal injective cogenerator for the category of $R_{S}$-modules, contrary to what is stated in the proof of [1, Lemma 2]:

Example 3.1. Let $(R, m)$ be a local ring of dimension at least two and $p$ any prime which is not maximal or minimal. By [4, Lemma 4.1], every element of Spec $R_{p}$ is an associated prime of the $R_{p}$-module $\operatorname{Hom}_{R}\left(R_{p}, E_{R}\right)$. In particular, $\operatorname{Hom}_{R}\left(R_{p}, E_{R}\right) \neq E_{R_{p}}$.

Example 3.2 ([4, p. 127]). Let $R$ be a local domain such that the completion of $R$ has a nonminimal prime contracting to $(0)$ in $R$. Let $Q$ be the field of fractions of $R$. Then $\operatorname{Hom}_{R}\left(Q, E_{R}\right)$ is not Artinian.

Example 3.3. Let $R$ be a Noetherian domain which is not local. Let $m \neq n$ be maximal ideals of $R$. By a slight modification of the proof of [4, Lemma 4.1], one obtains that (0) is an associated prime of $\operatorname{Hom}_{R}\left(R_{m}, E_{R}(R / n)\right)$, which is a direct summand of $\operatorname{Hom}_{R}\left(R_{m}, E_{R}\right)$. Hence, $\operatorname{Hom}_{R}\left(R_{m}, E_{R}\right) \nRightarrow E_{R_{m}}$.

We now show that the converse to part (a) of Theorem 1.1 does not hold in general. Let $R$ be a domain and $Q$ its field of fractions. Of course, $Q$ is reflexive as a $Q=R_{(0)}$-module. But as the following theorem shows, $Q$ is rarely a reflexive $R$-module.

Proposition 3.4. Let $R$ be a Noetherian domain and $Q$ the field of fractions of $R$. Then $Q$ is a reflexive $R$-module if and only if $R$ is a complete local domain of dimension at most one.

Proof. We first suppose $R$ is a one-dimensional complete local domain with maximal ideal $m$. Let $E=E_{R}(R / m)$. By [5. Theorem 2.5], $\operatorname{Hom}_{R}(Q, E) \cong Q$. Since the evaluation map of the Matlis double dual is always injective, we obtain that $Q \longrightarrow \operatorname{Hom}_{R}\left(\operatorname{Hom}_{R}(Q, E), E\right)$ is an isomorphism.

Conversely, suppose $Q$ is a reflexive $R$-module. By Theorem 2.1, $R$ is a complete semilocal domain, hence local. It suffices to prove that $\operatorname{dim} R \leq 1$. Again by Theorem 2.1, there exists a finitely generated $R$-submodule $N$ of $Q$ such that $Q / N$ is Artinian. Since $\operatorname{Ann}_{R} N=0, \operatorname{dim} R=\operatorname{dim} N$. Thus, it suffices to prove that $H_{m}^{i}(N)=0$ for $i \geq 2$. But this follows readily from the facts that $H_{m}^{i}(Q)=0$ for all $i$ and $H_{m}^{i}(Q / N)=0$ for $i \geq 1$ (as $Q / N$ is Artinian). 


\section{ACKNOWLEDGMENTS}

The authors would like to thank Peder Thompson for many helpful discussions on this topic. They are also very grateful to Bill Heinzer for pointing out the existence of Proposition 2.5 .

\section{REFERENCES}

[1] Richard G. Belshoff, Edgar E. Enochs, and Juan Ramon García Rozas, Generalized Matlis duality, Proc. Amer. Math. Soc. 128 (2000), no. 5, 1307-1312, DOI 10.1090/S0002-9939-9905130-8. MR1641645

[2] R. Berger, R. Kiehl, E. Kunz, and H.-J. Nastold, Differentialrechnung in der analytischen Geometrie (German), Lecture Notes in Mathematics, No. 38, Springer-Verlag, Berlin-New York, 1967. MR 0224870

[3] Craig Huneke and Irena Swanson, Integral closure of ideals, rings, and modules, London Mathematical Society Lecture Note Series, vol. 336, Cambridge University Press, Cambridge, 2006. MR:2266432

[4] Leif Melkersson and Peter Schenzel, The co-localization of an Artinian module, Proc. Edinburgh Math. Soc. (2) 38 (1995), no. 1, 121-131, DOI 10.1017/S0013091500006258. MR1317331

[5] Peter Schenzel, A note on the Matlis dual of a certain injective hull, J. Pure Appl. Algebra 219 (2015), no. 3, 666-671, DOI 10.1016/j.jpaa.2014.05.020. MR3279381

Department of Mathematics, University of Dallas, Irving, Texas 75062-4736

E-mail address: ddailey@udallas.edu

Department of Mathematics, University of Nebraska-Lincoln, Lincoln, Nebraska 68588-0130

E-mail address: tmarley1@unl.edu 